\title{
A priori postulated and real power in cluster randomized trials: mind the gap Lydia Guittet $^{1,2}$, Bruno Giraudeau*3,4 and Philippe Ravaud ${ }^{1,2}$
}

\author{
Address: ${ }^{1}$ Département d'Epidémiologie, Biostatistique et Recherche Clinique, Groupe Hospitalier Bichat-Claude Bernard (AP-HP) - Université \\ Paris 7, Paris, France, 2INSERM U 738, Université Paris 7, Paris, France, 3INSERM CIC 202, Faculté de Médecine, Université François Rabelais, \\ Tours, France and ${ }^{4}$ INSERM U 717, Université Paris 7, Paris, France \\ Email: Lydia Guittet - lguittet@free.fr; Bruno Giraudeau* - giraudeau@med.univ-tours.fr; Philippe Ravaud - philippe.ravaud@bch.ap-hop- \\ paris.fr \\ * Corresponding author
}

Published: 18 August 2005

BMC Medical Research Methodology 2005, 5:25 doi:10.1 186/147/-2288-5-

25

This article is available from: http://www.biomedcentral.com/I47I-2288/5/25

This is an Open Access article distributed under the terms of the Creative Commons Attribution License (http://creativecommons.org/licenses/by/2.0), which permits unrestricted use, distribution, and reproduction in any medium, provided the original work is properly cited.

\begin{abstract}
Background: Cluster randomization design is increasingly used for the evaluation of health-care, screening or educational interventions. The intraclass correlation coefficient (ICC) defines the clustering effect and be specified during planning. The aim of this work is to study the influence of the ICC on power in cluster randomized trials.

Methods: Power contour graphs were drawn to illustrate the loss in power induced by an underestimation of the ICC when planning trials. We also derived the maximum achievable power given a specified ICC.

Results: The magnitude of the ICC can have a major impact on power, and with low numbers of clusters, $80 \%$ power may not be achievable.

Conclusion: Underestimating the ICC during planning cluster randomized trials can lead to a seriously underpowered trial. Publication of $a$ priori postulated and a posteriori estimated ICCs is necessary for a more objective reading: negative trial results may be the consequence of a loss of power due to a mis-specification of the ICC.
\end{abstract}

\section{Background}

A cluster randomized trial involves randomizing social units or clusters of individuals, rather than the individuals themselves. This design, which is increasingly used for evaluating health-care, screening and educational interventions [1-3], presents specific constraints that must be considered during planning and analysis $[4,5]$.

The responses of individuals within a cluster tend to be more similar than those of individuals of different clusters. This correlation leads to an increased required sam- ple size in randomized trials of clusters compared with that of individuals, although this clustering effect is rarely taken into account. Thus, in a recent review of cluster randomized trials in primary care, Eldridge et al [6] reported that only $20 \%$ of studies accounted for clustering in the sample size calculation. Similar results were found in other reviews, as listed by Bland [7]. The increase in sample size is measured through an inflation factor, which is a function of both the cluster size and the intraclass correlation coefficient (ICC), which appraises the correlation between individuals within the same cluster $[1-3,8]$. 
Therefore an a priori value for this correlation must be postulated during planning. However, estimates of this correlation are rarely available, and, if available, are often uncertain. Indeed the correlation would differ according to outcome, setting, intervention, covariate adjustment and also sampling $[5,9,10]$. Therefore, a discrepancy between $a$ priori postulated and $a$ posteriori estimated ICCs may occur.

The discrepancy between $a$ priori postulated and a posteriori estimated ICCs may be reduced by intermediate estimation of the ICC, thus allowing a re-estimation of the required sample size [11]. However, the room to manœuvre to increase the sample size may be restricted. Indeed, including new clusters may be difficult, either because the number of clusters is limited [10,12-15] (which may occur when the randomization unit is defined by a geographic area or hospital, for example) or because clusters are frequently randomized all at once and not one at a time. The cluster size itself may also be limited (e.g., by the size of a family or because the number of patients followed up in a clinical practice cannot be increased [16]), which then disallows the increase in sample size by increasing cluster size.

The purpose of our study was to assess the consequence on power of the ICC and to what extent the discrepancy between $a$ priori postulated and $a$ posteriori estimated ICCs may induce a loss in power in cluster randomized trials.

\section{Methods}

We considered a completely cluster randomized design with a continuous outcome (normally distributed) measured at a single time point. We assumed an equal number of clusters randomized to each arm and a fixed common cluster size. The sample size is calculated as follows [1]:

$$
m g=\frac{2 \sigma^{2}[1+(m-1) \rho]\left(z_{1-\alpha / 2}+z_{1-\beta}\right)^{2}}{\Delta^{2}}=\frac{2[1+(m-1) \rho]\left(z_{1-\alpha / 2}+z_{1-\beta}\right)^{2}}{E S^{2}}
$$

where $m$ is the cluster size, $g$ is the number of clusters per arm, $\rho$ is the ICC, ES is the effect size (defined as ratio between the absolute difference between the two intervention-specific means $(|\Delta|)$ and the standard deviation $(\sigma))$ and $z_{1-\alpha / 2}$ and $z_{1-\beta}$ are the critical values of the standard normal distribution corresponding to error rates $\alpha$ (two-sided) and $\beta$, respectively. One recognizes the sample size calculation for an individually randomized trial inflated by a factor equal to $[1+(m-1) \rho]$ defined as the variance inflation factor. When the cluster size varies, $m$ refers to the average cluster size.

\section{Power contour graphs}

To quantify the influence of the ICC on the power, we drew two kinds of power contour graphs. First, consider- ing an effect size and an a priori postulated ICC, we considered several combinations of numbers of clusters and cluster sizes that allow for achieving $80 \%$ power. Then considering these combinations, we plotted the real power as a function of the ICC, which may differ from the a priori postulated value. Two values of a priori postulated ICC $(0.005,0.02)$ and five numbers of clusters per intervention arm $(3,5,10,20$ and 40) were considered for these graphs. The effect size was fixed at 0.25 .

We also drew power contour graphs, showing combinations of cluster sizes and number of clusters leading to a pre-specified power, with type I error fixed at 5\%. Four power levels were considered $(90,80,60$ and 40\%), 3 effect sizes $(0.25,0.50$ and 0.75$)$ and 4 levels of $\rho(0.005$, $0.020,0.050$ and 0.100 ). These ICC values were chosen according to previously published estimates $[3,6,12,16$ 23].

\section{Maximal theoretical achievable power}

We determined the maximal achievable power given a limited number of randomized clusters (i.e., considering an infinite cluster size) or a limited cluster size (i.e., considering an infinite number of clusters). For a limited number of clusters, results were graphically illustrated by considering 5 numbers of clusters per intervention arm (3, $5,10,20$ and 40$)$ for 2 effect sizes $(0.25,0.5)$.

\section{Results \\ Influence of the discrepancy between a priori postulated and a posteriori estimated ICCs on power}

Figure 1 displays the real power associated with a study whose $a$ posteriori estimated ICC would differ from the $a$ priori postulated one. With an a priori ICC of 0.02, as few as 5 clusters per intervention arm is not enough to achieve a power of $80 \%$ to detect an effect size of 0.25 . Power decreases as the ICC increases, and the loss is all the more important when the number of clusters is small. For example, if the a priori ICC was fixed at 0.005 and the $a$ posteriori ICC is as high as 0.01 , the power falls to $70.8 \%$ with 5 clusters per intervention arm, instead of the targeted $80 \%$ power, whereas the power is almost safeguarded with 20 clusters per intervention arm (real power $77.7 \%)$.

Figure 2 displays power contour graphs for combinations of numbers of clusters and cluster sizes. First, let us consider the situation of a fixed number of clusters. In many situations, even a slight increase in the ICC has a great influence on power and leads to a major increase in the required cluster size to keep the desired power. In some situations, even reaching the required power may no longer be possible: the power contour curves tend to be infinite. For instance, assuming that 15 clusters are randomized to each arm and we want to detect an effect size of 


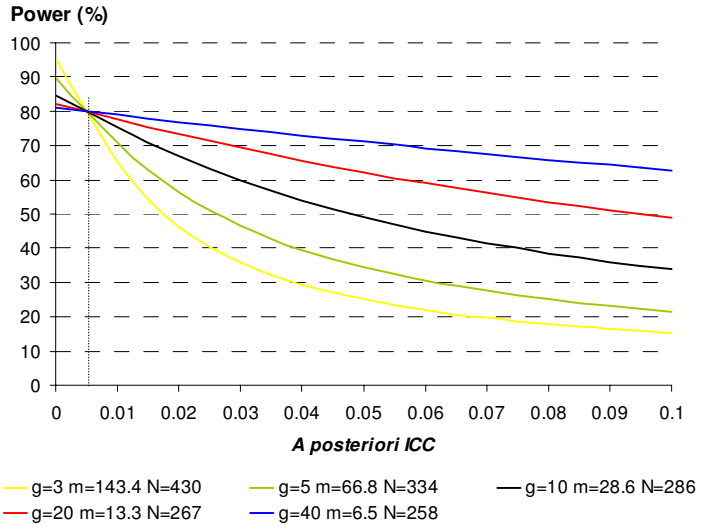

A priori ICC $=0.005$

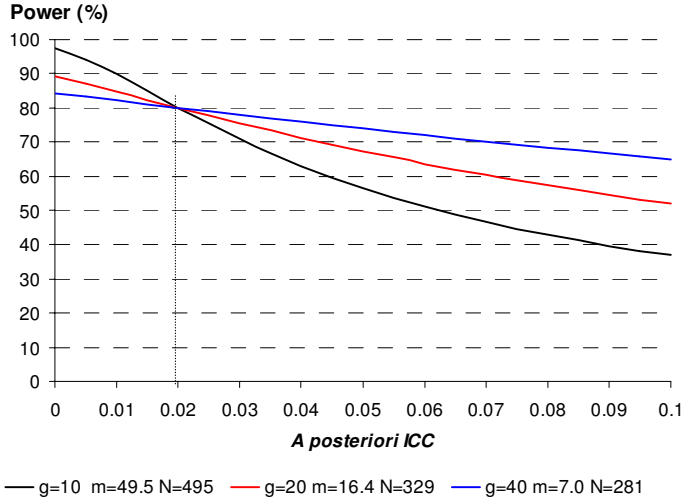

A priori ICC $=0.02$

\section{Figure I}

Real power of cluster randomized trials according to the discrepancy between the a priori postulated and a posteriori estimated intraclass correlation coefficients. The effect size to be detected is fixed at 0.25 and power at $80 \%$. $g$ is the number of clusters per arm, $m$ is the average cluster size and $N$ is the total number per intervention arm considering an $a$ priori postulated ICC of 0.005 or 0.02 .

0.25 with $80 \%$ power, we would need an average cluster size of 25 patients with an a priori ICC fixed at 0.02 . To keep $80 \%$ power, the required mean cluster size should be increased to 98 for an ICC of 0.05, which represents 1095 more subjects per arm. If the ICC actually equals 0.10 , $80 \%$ power is no longer achievable without recruiting additional clusters. The phenomenon is all the more acute when the number of fixed clusters is low.

Second, when the mean cluster size is limited but the number of clusters is not, an increase in the ICC may also be of great consequence. As an example, considering a mean cluster size of 100 , we would need to randomize 8 clusters per arm to detect a 0.25 effect size with $80 \%$ power when the ICC is fixed at 0.02 . This number of clusters is raised to 15 and 28 when the ICC is fixed at 0.05 and 0.10 , respectively, or 700 and 2000 additional subjects, respectively, per arm.

\section{Maximal theoretical power with infinite cluster size}

In a cluster randomized trial aimed at detecting an effect size ES at a pre-specified $\alpha$ level with an a priori postulated ICC equal to $\rho$, changing the cluster size $m$ and/or the number of clusters $g$ per group changes $\beta$ and therefore power. Power is thus related to the $f(m, g)=\left(z_{1-\alpha / 2}+z_{1-\beta}\right)^{2}$ function defined as $f(m, g)=\frac{m g E S^{2}}{2[1+(m-1) \rho]}$.

When $m$, the mean cluster size, tends to be infinite, $f(m, g)$ tends to be an asymptotic value, but there is no limit when $g$, the number of clusters, is infinite:

$$
\lim _{m \rightarrow+\infty} f(m, g)=\frac{g E S \dagger}{2 \rho} \quad \lim _{g \rightarrow+\infty} f(m, g)=+\infty
$$

Therefore, although power is not theoretically limited when the number of clusters can be increased, a maximal reachable power is possible when this number is fixed and only the cluster size can be increased. This maximum theoretical power is defined as:

Power $=\Phi^{-1}\left(\sqrt{\frac{g E S^{2}}{2 \rho}}-z_{1-\alpha / 2}\right)$

where $\Phi^{-1}()$ refers to the inverse cumulative function associated with the standard normal distribution. This maximal theoretical power decreases when the ICC increases and/or the number of clusters decreases (Figure 3 ). In some cases, an $80 \%$ or $90 \%$ power is not achievable even with a theoretical situation of infinite cluster sizes. 

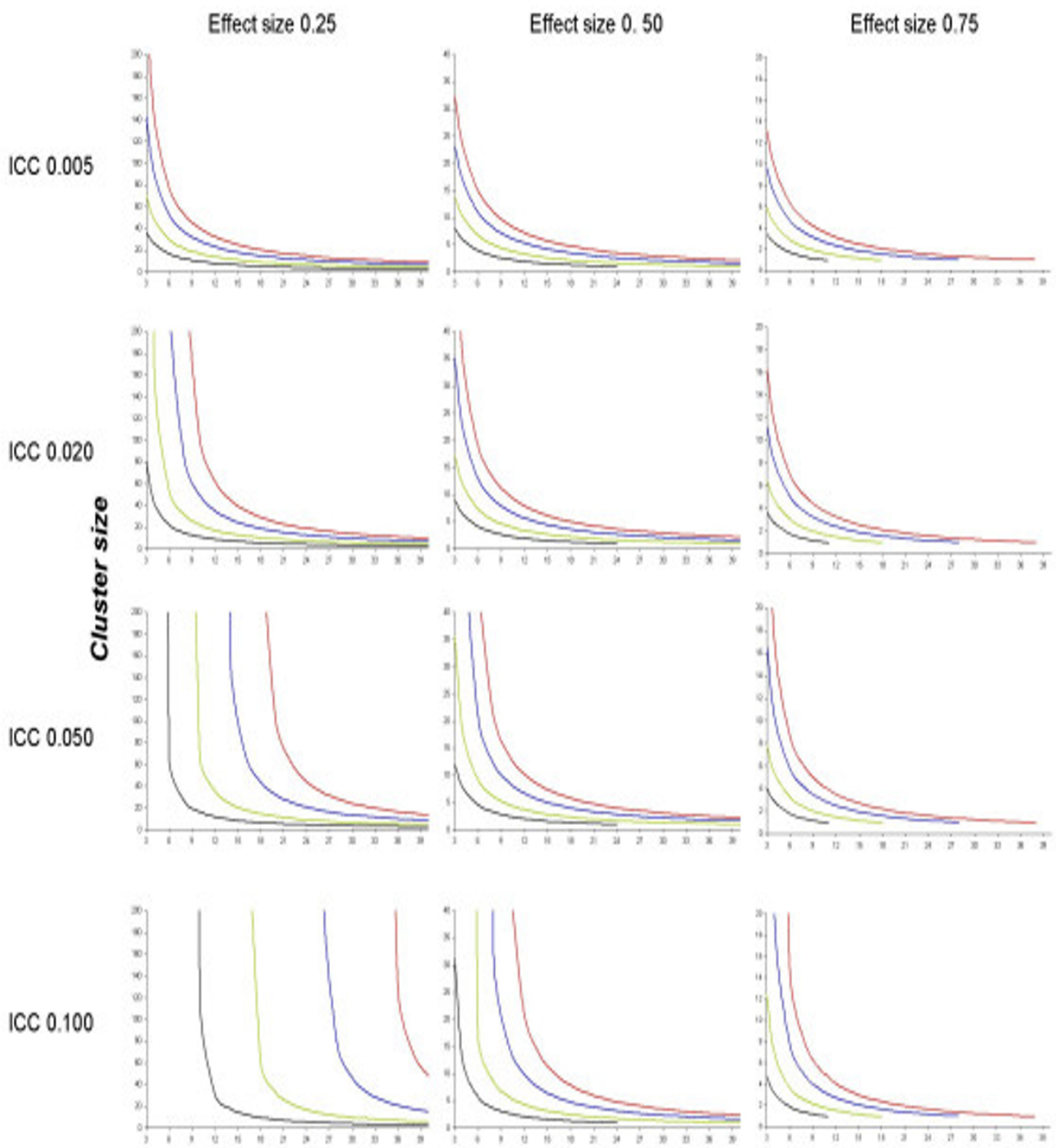

\section{Number of clusters per intervention arm}

$90 \%$ power $80 \%$ power $60 \%$ power $40 \%$ power

\section{Figure 2}

Power contour graphs for several intraclass correlation coefficients (ICCs) and effect sizes*. Effect size is presented in columns and ICC in rows. In situations above or to the right of the red curve, the statistical power is greater than $90 \%$. In situations between the red and blue curves, the statistical power is between $80 \%$ and $90 \%$. In situations between the blue and red curves, the statistical power is between $60 \%$ and $80 \%$. For vertical curves, increasing the cluster size is pointless. The number of subjects required, assuming individual randomization, is 24 to achieve a power of $40 \%$ to detect an effect size of 0.50 , and 38,28 , I 8 and II to achieve powers of $90 \%, 80 \%, 60 \%$ and $40 \%$, respectively, to detect an effect size of 0.75 , thus, the reason why curves are truncated. *Effect size $=$ absolute difference between the two intervention-specific means divided by the S.D. of the response variable. 


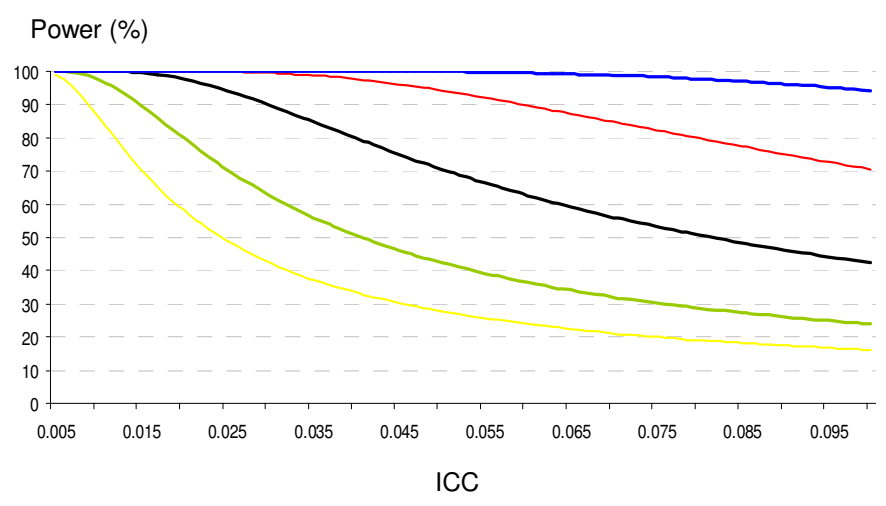

Effect size $=0.25$

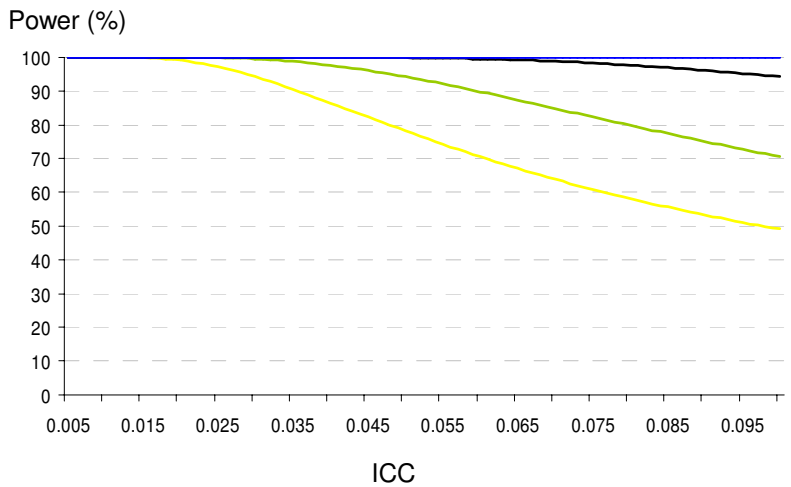

Effect size $=0.50$

\section{Figure 3}

Theoretical maximal power assuming an infinite cluster size for several fixed numbers of clusters according to two different effect sizes *. *Effect size $=$ absolute difference between the two intervention-specific means divided by the S.D. of the response variable.

Thus, when 5 clusters are randomized to each arm, a power of $80 \%$ to detect an effect size of 0.50 cannot be achieved if the ICC is greater than 0.079 (and this limit equals 0.058 when $90 \%$ power is considered). For an effect size of 0.25 , this upper ICC limit is 0.019 for $80 \%$ power and 0.014 for $90 \%$ power.

\section{Discussion}

The ICC is a nuisance parameter that has to be a priori specified when planning a cluster randomized trial. The magnitude of this coefficient has a major impact on power, particularly with a small number of randomized clusters. Our results were derived considering a continuous outcome, but in their simulation study, Donner and Klar [24] showed that power never differs from more than one percentage point in continuous or binary outcomes. Moreover, we did not take into account any potential variability in cluster size, which is already known to reduce power [25]. When planning cluster randomized trials, variability in cluster size is rarely taken into account, and the cluster size $m$ is generally replaced by the mean cluster size. An underestimation of the ICC may therefore be expected to have similar consequences when cluster size is constant. In the end, an underestimation of the ICC during planning could therefore lead to a severely underpowered study and thus questionable results.

In cluster randomized trials, it is known that for a fixed total number of subjects, the higher the number of clus- ters (and thus the smaller the average cluster size), the higher the power $[2,4,5,14,24,26,27]$. In the extreme case, in clusters of size one, individuals are randomized, with no loss of power because of correlation between subjects. Moreover, it has also been shown that increasing cluster size improves the power up to a certain threshold, which depends on the value of the ICC $[24,27]$. Therefore, when planning a cluster randomized trial, the optimal strategy is indeed to randomize a large number of clusters $[1,2,12,29]$. Such a strategy first allows for decreasing the total sample size for a pre-specified power and second, as our results show, protects against a loss of power induced by an underestimation of the ICC when planning. However, because of logistic constraints, the number of randomized clusters may be limited, and indeed, the review by Eldridge et al [6] noted that half of the cluster randomized trials analyzed had fewer than 29 clusters in each arm. Therefore, for most cluster randomized trials, the a priori postulated value of the ICC has a great impact on power.

When planning trials, the a priori postulated ICC will rarely be very reliable. During the study, an intermediate estimation of the ICC can be assessed, thus allowing a sample size adjustment [11]. But the determination of this intermediate estimation is not without error, as was shown in the study by Moore et al [28], in which the intermediate ICC was 0.012 and the final one 0.031 . A sensitivity analysis must therefore be undertaken when planning, to account for uncertainty of the ICC. In the 
extreme situations, when very few clusters can be randomized, such a sensitivity analysis may illustrate the high risk of performing an underpowered study and thus highlight arguments for not performing the study.

When reporting the study results, investigators should publish both the ICC used during the planning and the $a$ posteriori estimated one, as recommended initially by some authors and recently by the extension of the CONSORT statement for cluster randomized trials [27,29-31]. However, such information is rarely available. We studied cluster randomized trials published between January 2003 and December 2004 in the British Medical Journal, "which contains more such reports than any other journal" [7], and the published extension of the CONSORT statement [30]). Of 16 published studies, 5 (31.2\%) did not report an a priori postulated ICC and 2 reported no sample size calculation. Only 5 (31.2\%) reports provided a posteriori estimated ICCs (without any confidence intervals). Such under-reporting disallows assessing the discrepancy between the a priori postulated ICC and the $a$ posteriori estimated one. However, reporting both ICCs would help readers "assess the appropriateness of the original sample size calculations as well as the magnitude of the clustering for each outcome" [30] and help investigators design future trials $[1,27,31]$. It would also help readers understand trial results, particularly negative ones: a study may prove to be negative just by a loss of power induced by an $a$ priori underestimation of the ICC. On a formal point, the publication format of the a posteriori estimated ICC should follow the recommendation by Campbell et al., who advocate specifying a description of the data set and information on the method used to assess it and the precision of the estimate [32].

In conclusion, our study supports modifications in investigators' practices when planning trials and reporting results, taking into account the uncertainty of the ICC by favoring a high number of clusters and publishing this parameter. For readers, an objective reading of trial results, particularly negative results, requires knowledge of $a$ priori and $a$ posteriori estimated ICCs.

\section{Competing interests}

The author(s) declare that they have no competing interests.

\section{Authors' contributions}

This study was designed by LG, BG and PhR. LG performed the statistical analysis and drafted the article, which was then revised by BG and PhR.

\section{Acknowledgements}

The authors are indebted to Dr. Sandra Eldridge for constructive comments.
This work was funded by a grant from the Foundation for Medical Research (FRM).

\section{References}

I. Donner A, Klar N: Design and Analysis of Cluster Randomization Trials in Health Research London, England: Arnold; 2000.

2. Murray DM: Design and Analysis of Group-Randomized Trials New York, NY: Oxford University Press Inc; 1998.

3. Reading R, Harvey I, McLean $M$ : Cluster randomised trials in maternal and child health: implications for power and sample size. Arch Dis Child 2000, 82:79-83.

4. Murray DM, Varnell SP, Blitstein JL: Design and analysis of grouprandomized trials: a review of recent methodological developments. Am J Public Health 2004, 94:423-432.

5. Donner A, Klar N: Pitfalls of and controversies in cluster randomization trials. Am J Public Health 2004, 94:4I6-422.

6. Eldridge SM, Ashby D, Feder GS, Rudnicka AR, Ukoumunne OC: Lessons for cluster randomized trials in the twenty-first century: a systematic review of trials in primary care. Clinical trials 2004 , I:80-90.

7. Bland JM: Cluster randomised trials in the medical literature: two bibliometric surveys. BMC Med Res Methodol 2004, 4:2I.

8. Kerry SM, Bland JM: Statistics notes: sample size in cluster randomisation. $B M]$ I 998, 3 I 6:549.

9. Adams G, Gulliford MC, Ukoumunne OC, Eldridge S, Chinn S, Campbell MJ: Patterns of intra-cluster correlation from primary care research to inform study design and analysis. J Clin Epidemiol 2004, 57:785-794.

10. Turner RM, Prevost AT, Thompson SG: Allowing for imprecision of the intracluster correlation coefficient in the design of cluster randomized trials. Stat Med 2004, 23: | | $95-12$ | 4.

II. Lake S, Kammann E, Klar N, Betensky R: Sample size re-estimation in cluster randomization trials. Stat Med 2002 , 21:1337-1350.

12. Ukoumunne OC, Gulliford MC, Chinn C, Sterne JAC, Burney PG]: Methods for evaluating area-wide and organisation-based interventions in health and health care: a systematic review. Health Technology Assessment (Winchester, England) 1999, 3:iii-92.

13. Ukoumunne OC, Gulliford MC, Chinn S, Sterne JAC, Burney PGJ, Donner A: Evaluation of health interventions at area and organisation level. BM] 1999, 3 19:376-379.

14. Flynn TN, Whitley E, Peters TJ: Recruitment strategies in a cluster randomized trial-cost implications. Stat Med 2002 2I:397-405.

15. Campbell MK, Thomson S, Ramsay CR, MacLennan GS, Grimshaw JM: Sample size calculator for cluster randomized trials. Comput Biol Med 2004, 34: I I 3-25.

16. Gulliford MC, Adams G, Ukoumunne OC, Latinovic R, Chinn S, Campbell MJ: Intraclass correlation coefficient and outcome prevalence are associated in clustered binary data. J Clin Epidemiol 2005, 58:246-25I.

17. Hannan PJ, Murray DM, David RJ, Mc Govern PG: Parameters to aid in the design and analysis of community trials: intraclass correlations from the Minnesota Heart Health Program. Epidemiology 1994, 5:88-95.

18. Siddiqui O, Hedeker D, Flay BR, Hu FB: Intraclass correlation estimates in a school-based smoking prevention study. Am Epidemiol 1996, I 44:425-433.

19. Smeeth L, Siu-Woon Ng E: Intraclass correlation coefficients for cluster randomized trials in primary care: data from the MRC Trial of the Assessment and Management of Older People in the Community. Control Clin Trials 2002, 23:409-42I.

20. Martinson BC, Murray DM, Jeffery RW, Hennrikus DJ: Intraclass correlation for measures from a worksite health promotion study: estimates, correlates, and applications. Am J Health Promot 1999, 13:347-357.

21. Murray DM, Phillips GA, Birnbaum AS, Lytle LA: Intraclass correlation for measures from a middle school nutrition intervention study: estimates, correlates, and applications. Health Educ Behav 200I, 28:666-679.

22. Murray DM, Clark MH, Alexander CW: Intraclass correlation from a community-based alcohol prevention study: the effect of repeat observations on the same communities. J Stud Alcohol 2000, 61:881-890. 
23. Murray DM, Short BJ: Intraclass correlation among measures related to tobacco use by adolescents: estimates, correlates, and applications in intervention studies. Addictive behaviours 1997, 22:1-12

24. Donner A, Klar N: Statistical considerations in the design and analysis of community intervention trials. J Clin Epidemiol 1996, 49:435-439.

25. Manatunga AK, Hudgens MG, Chen S: Sample size estimation in cluster randomized studies with varying cluster size. Biom J 200I, I:75-86.

26. Kerry SM, Bland JM: Unequal cluster sizes for trials in English and Welsh general practice: implications for sample size calculations. Stat Med 200I, 20:377-390.

27. Donner A: Some aspects of the design and analysis of cluster randomization trials. Appl Statistics 1998, 47:95-II3.

28. Moore L, Campbell R, Whelan A, Mills N, Lupton P, Misselbrook E, Frohlich J: Self help smoking cessation in pregnancy: cluster randomised controlled trial. BM] 2002, 325:| 383-| 388 .

29. Murray DM, McKinlay SM, Martin D, Donner AP, Dwyer JH, Raudenbush SW, Graubard BI: Design and analysis issues in community trials. Evaluation Review 1994, 18:493-5।4.

30. Campbell MK, Elbourne DR, Altman DG: CONSORT statement: extension to cluster randomised trials. BMJ 2004, 328:702-708.

31. Elbourne DR, Campbell MK: Extending the CONSORT statement to cluster randomized trials: for discussion. Stat Med 200I, 20:489-496.

32. Campbell MK, Grimshaw JM, Elbourne DR: Intracluster correlation coefficients in cluster randomized trials: empirical insights into how they should be reported. BMC Med Res Methodol 2004, 4:9.

\section{Pre-publication history}

The pre-publication history for this paper can be accessed here:

http://www.biomedcentral.com/1471-2288/5/25/prepub

Publish with Biomed Central and every scientist can read your work free of charge

"BioMed Central will be the most significant development for disseminating the results of biomedical research in our lifetime. "

Sir Paul Nurse, Cancer Research UK

Your research papers will be:

- available free of charge to the entire biomedical community

- peer reviewed and published immediately upon acceptance

- cited in PubMed and archived on PubMed Central

- yours - you keep the copyright 